\title{
53-Line Multi-wavelength Generation in Brillouin/Erbium Fiber Laser with Enhanced Stokes Feedback Coupling
}

\author{
Kap-Dong Park, Jae-Hyoung Park*, Namkyoo Park*, Jai-Hyung Lee, and Joon-Sung Chang \\ Laser and Quantum Optics Lab., Dept. of Physics, Seoul National University, Seoul, Korea \\ Tel. : +82-2-871-2861,Fax. : 82-2-884-3002 \\ E-mail:kdpark@photon.snu.ac.kr, jailee@photon.snu.ac.kr, jschang@photon.snu.ac.kr \\ Optical Communication Systems Laboratory, School of EE, Seoul National University, Seoul, Korea* \\ Tel: +82-2-880-1820, Fax:+82-2-885-5284,E-mail: jhpark@stargate.snu.ac.kr, nkpark@plaza.snu.ac.kr
}

We demonstrate a novel laser structure which enable efficient utilization of the laser gain profile and recirculation of Brillouin shifted signals inside the cavity. Multi-wavelength operation of more than 53 Stokes lines has been obtained with excellent flatness and stability. Procedures for optimum operation conditions will be described. 


\title{
53-Line Multi-wavelength Generation of Brillouin/Erbium Fiber Laser with Enhanced Stokes Feedback Coupling
}

\author{
Kap-Dong Park, Jae-Hyoung Park*, Namkyoo Park*, Jai-Hyung Lee, and Joon-Sung Chang \\ Laser and Quantum Optics Lab., Dept. of Physics, Seoul National University, Seoul, Korea \\ Tel. : +82-2-871-2861,Fax. : 82-2-884-3002 \\ E-mail:kdpark@photon.snu.ac.kr, jailee@photon.snu.ac.kr, jschang@photon.snu.ac.kr \\ Optical Communication Systems Laboratory, School of EE, Seoul National University, Seoul, Korea* \\ Tel: +82-2-880-1820, Fax: +82-2-885-5284, E-mail: jhpark@stargate.snu.ac.kr, nkpark@ plaza.snu.ac.kr
}

\section{Introduction}

There have been increased research efforts for Multi-channel laser sources, to meet the possible demand from the wavelength multiplexed sensor/communication systems. Densely shifted wavelength spacing and stabilized/flattened peak powers between neighbor channels are two key performance parameters for real interest. In view of power consumption aspects, operation with low threshold is also important.

Different approaches have been addressed in former studies [1 - 5] to fulfill above requirements, including a Brillouin fiber lasers. Of these, a hybrid Brillouin/Erbium fiber laser (BEFL) with feedback scheme is one of the strong candidates for realizing ultra dense wavelength operation. Recent studies followed different approaches in achieving feedback of higher order Stokes signals into the BEFL cavity, but were identical in its basic operation principle. The latest evolution was by D. Yu's 30 cahnnel operation with internal reflecting feedback circuit with a circulator and reflecting mirror [3], following Kim et al.'s 4 line operation with feedback coupler [4] and Cowle's 12 line operation [5].

Still the results demonstrated so far, exhibited limitations in the number of laser lines, output power stability, and flatness in channel peak powers. In this paper we demonstrate more than 53 lasing lines through optimum balancing of cavity gain profile and Brillouin injection signal, inside a revised cavity structure for effective feedback coupling and pump efficiency. We also describe the optimization algorithm and inter-relations between key parameters for the stable operation of the BEFL.

\section{Experimental Setup and Results}

Fig. 1 shows the schematic diagram of revised BEFL structure with internal feedback, used in this study. Single mode optical coupler (SMOC1) with 3dB coupling ratio was used to inject Brillouin Pump into the main cavity. $10 \%$ optical coupler (SMOC2), isolator, and polarization controllers (PCs) were also included inside the cavity to provide the feedback Stokes signal to the $10 \mathrm{~km}$ of single mode fiber (SMF) for next order Stokes generation, to enable unidirectional operation of the laser, and to control the polarization state of the cavity respectively. In order to increase the number of comb lines, EDF2 was also placed in the feedback loop. To enhance the efficiency of feedback coupling into the Brillouin medium while maintaining the power availability in the main loop, we placed an optical circulator to couple amplified Stokes feedback signal into the main cavity. Brillouin gain then accurately determines the Stokes wavelength separations while combined gain from EDF1 and EDF2 determine the envelope of the frequency comb.

To optimize the BEFL operation, we first checked inter-relationships between Brillouin pump power, EDFA output power in the main loop and EDFA output power in the feedback part. At zero Brillouin pump and fixed main-loop EDFA power, we observed ASE spectrum in the output port, becoming unstable in wider spectral range as we increased sub-loop EDFA power above some critical value. By adjusting main-loop pump power in this unstable operation regime, it was possible to get both wide ASE spectrum and good stability. This existence of stable operation point for broad ASE generation from the 


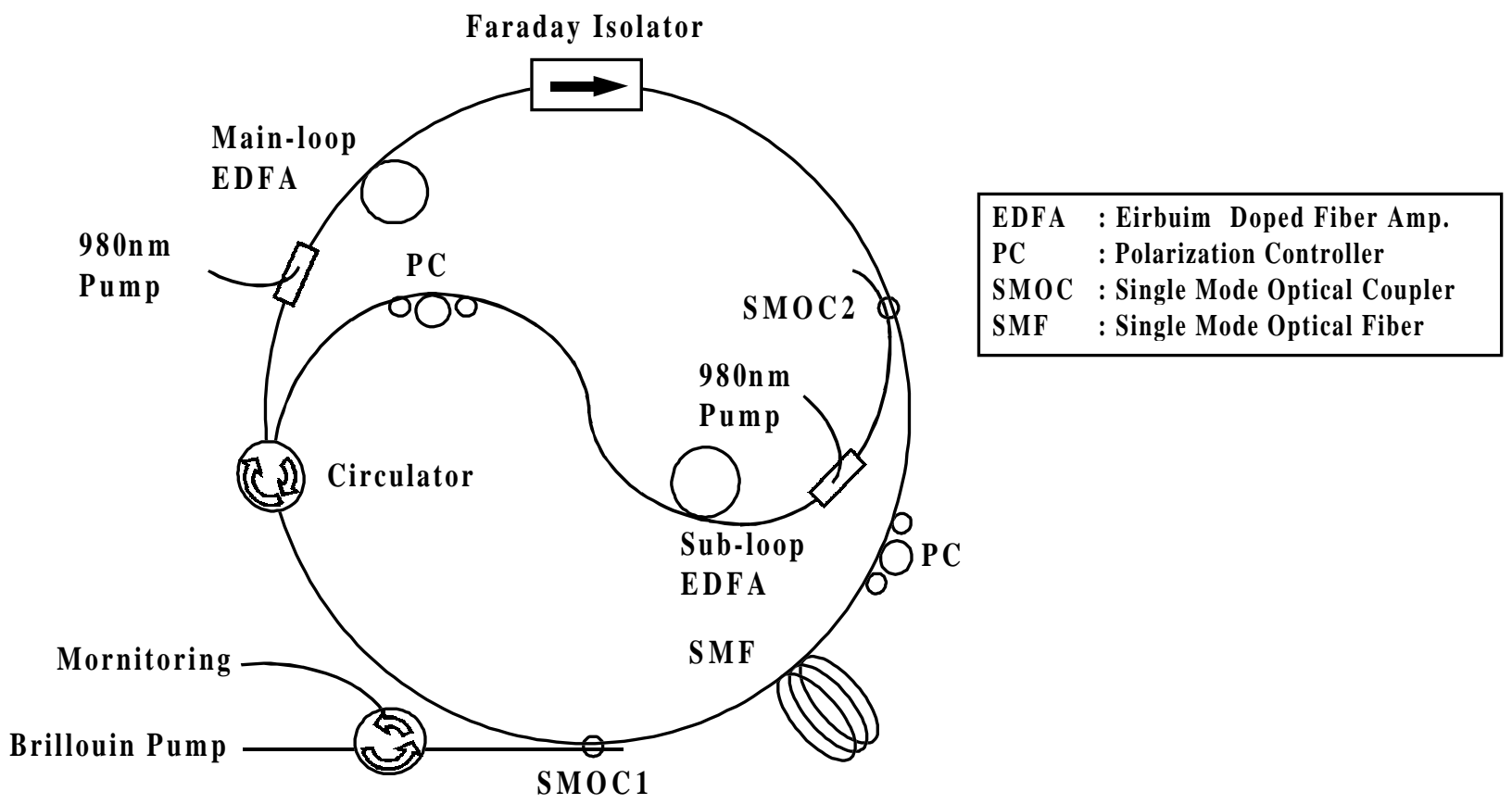

Fig.1. Schematic Diagram of BEFL with internal feedback

cavity without the Brillouin pump injection, plays a crucial role in achieving multiple generation to the higher order Stokes lines over wide spectral range.

We then injected Brillouin pump into the cavity to see the injection pump power dependence to the number of lasing lines at the maximum pump powers of main-loop and sub-loop EDFA, within stable, broad ASE generation regime as mentioned above. Fig.2 (A) illustrates the output spectrum of BEFL under small Brillouin pump power injection. With small injection pump signal, generated Stokes lines were not strong enough to suppress the ASE super-luminescence, ceasing the generation of the higher order Stokes lines beyond 1556nm. On the contrary, too large Brillouin pump injection depleted the photons in the cavity not providing enough gains to the higher order Stokes lines, as can be seen in Fig. 2 (B). In this case, lower spectral part of ASE super-luminescence was comparably suppressed by strong Brillouin pump power. The optimum balancing was achieved between these two limiting cases, under appropriate polarization controller settings. The absolute injection pump power for this stable operation showed dependency on the EDF gain and pump powers. For the maximum EDF gains, we observed
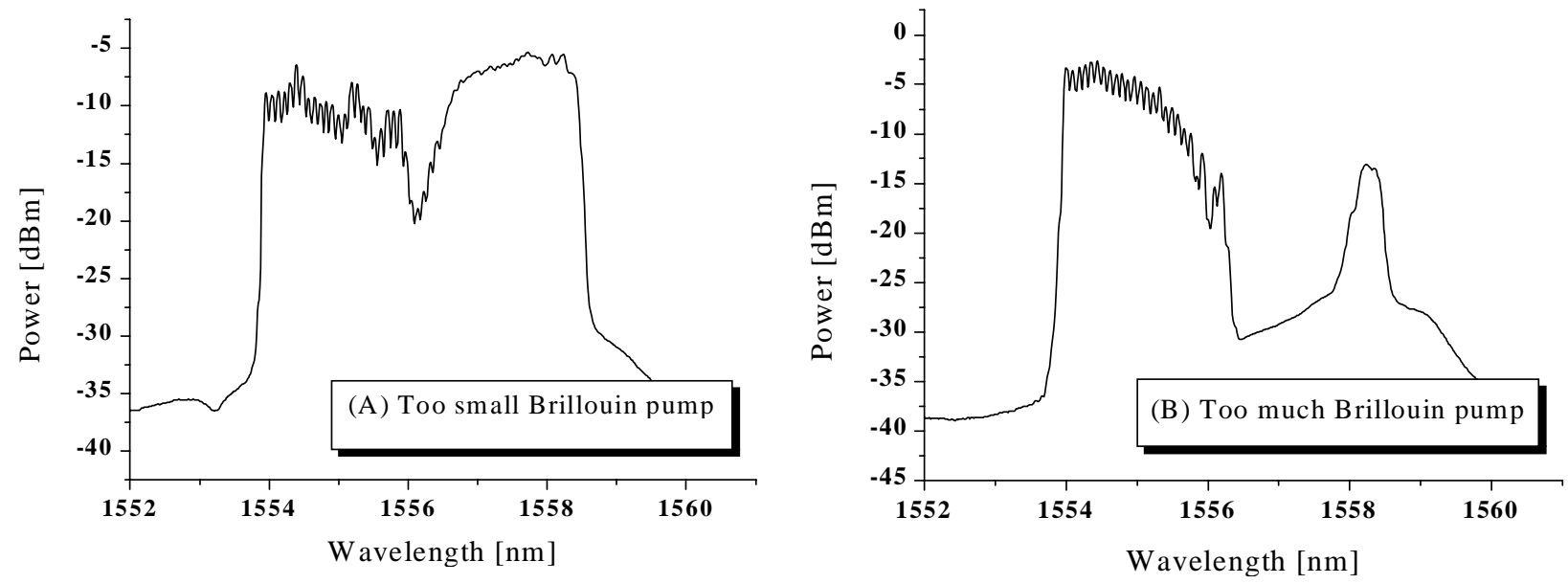

Fig.2. Comb output as a function of BP power 
injection pump power window between within a small power range over 1.50 2.17 mW. Since EDFA inversion efficiency changes as Brillouin pump power input changes, minor optimization process should be followed repeatedly whenever we increase Brillouin pump power. Fig.3 illustrates the optimized wavelength comb output spectrum, with highly increased number of comb lines. Some amount of power fluctuation was observed above $53^{\text {rd }}$ Stokes line while good stability was ensured below. It was also possible to get excellent flatness of peak power within a dB and stability of Stokes lines up to $43^{\text {rd }}$ order, at the expense of several higher order Stokes lines.

We also observed linear dependence between the number of Stokes lines and feedback pump power within stable operation regime, which can be readily expected from the cavity structure. This was done at the optimized EDF pumping power, Brillouin pump power and Brillouin wavelength. By optimizing all the parameters mentioned above and adjusting input wavelength, it was possible to obtain the optimum balance in Fig. 3 over nearly 5nm range.

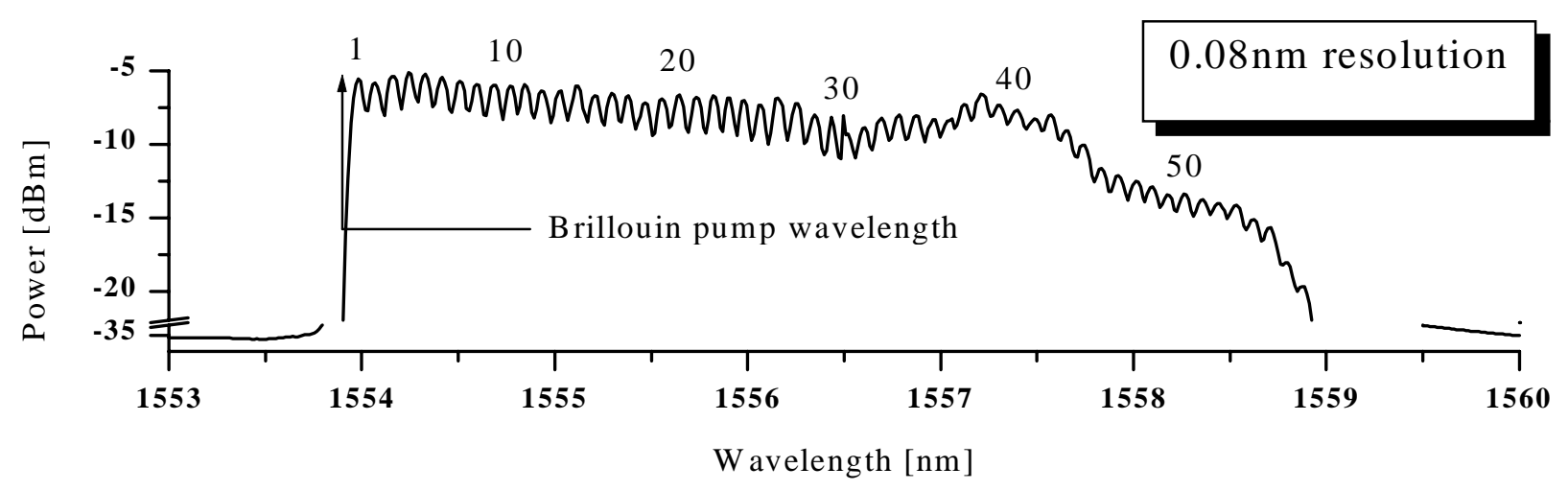

Fig.3. Comb output after optimization

\section{Conclusion}

We demonstrated 53-line multiple wavelength comb through improved cavity design in conjunction with the EDF gain and injected Brillouin pump power optimization. EDFA power balancing, injection power and polarization control were key parameters in maximizing the number of comb lines. More lines with decreased power variation will be expected through enhancing the polarization control and balancing the combined EDFA pumping at higher power level. We also described algorithm for achieving the optimum operation conditions. We believe this technique to be applicable to other types of lasers operating under the same basic principles.

\section{References}

[1] K. O. Hill, et al.: 'CW generation of multiple stokes and anti-stokes brillouin-shifted frequencies', Appl. Phys. Lett., vol. 29, pp. 185-187, 1976.

[2] N. Park, et. al.: '24 Lasing Line Multifrequency Operation of Fiber Ring Laser.', IEEE Photonics Technology Letters, vol. 8, pp. 1459-1461, 1996.

[3] D. Yu Stepanov and G.J. Cowle : ‘ 30-channel 10-GHz laser comb from a multiline Brillouin/erbium fiber laser', CLEO '97, May 1997, Paper CtuG6, pp.82

[4] N. S. Kim : 'Multiwavelength operation of EDFA-enhanced Brillouin/Erbium fiber lasers',Elecron. Lett., 1998, Vol.34, pp. 673-675

[5] G.J. Cowle et al.: ' Multiwavelength operation of Brillouin/Erbium fiber laser with injection-locked seeding', OFC '97, Feb. 1997, Paper TuH7, pp. 34-35 